\title{
Propuesta de proceso para la fabricación de probetas a base de caucho reciclado granulado
}

\section{Process proposal for the manufacture of test tubes based on granulated recycled rubber}

\author{
Jorge Fadul ${ }^{1}$, Ana Bernal ${ }^{2}$, Javier Batista ${ }^{1}$, Nacarí Marín ${ }^{3 *}$ \\ ${ }^{1}$ Licenciatura en Ingeniería Electromecánica, Facultad de Ingeniería Eléctrica, Universidad Tecnológica de Panamá, Sede \\ Azuero \\ ${ }^{2}$ Licenciatura en Ingeniería Aeronáutica. Facultad de Ingeniería Mecánica, Universidad Tecnológica de Panamá, Sede Azuero \\ ${ }^{3}$ Facultad de Ingeniería Mecánica, Universidad Tecnológica de Panamá, Sede Azuero
}

\begin{abstract}
Resumen El caucho es un material utilizado en diferentes aplicaciones debido a sus propiedades mecánicas, particularmente, las elásticas. Los niveles de contaminación a causa del elastómero son altos y van en aumento, de un $10 \%$ a un $28 \%$ durante la última década. La elaboración de productos a base de caucho reciclado representa una alternativa a la reducción de esta contaminación. Previo a la evaluación del potencial de reutilización del nuevo material a base de caucho, se requiere de la definición de probetas con formas y dimensiones específicas, basadas en normas estandarizadas. En este documento se presenta una propuesta de proceso para la elaboración de probetas cilíndricas y en forma de placas, a base de caucho reciclado granulado CRG y 7 aglomerantes comerciales seleccionados. Como resultado de esta investigación, las probetas obtenidas con sellador elastomérico de poliuretano y con adhesivo de poliuretano mantuvieron la forma al retirar la compresión y el molde. En un trabajo posterior, estas probetas serán sometidas a pruebas mecánicas que permitan determinar las propiedades mecánicas correspondientes.
\end{abstract}

Palabras clave Aglomerantes, caucho, propiedades químicas, propiedades térmicas, reciclaje.

\begin{abstract}
Rubber is a material used in different applications due to its mechanical properties, particularly elastic ones. Pollution levels from this elastomer are high and, on the rise, from $10 \%$ to $28 \%$ over the last decade. The production of products based on recycled rubber represents an alternative to reducing such pollution. Prior to evaluating the reuse potential of the new rubber-based material, it is required to define specimens with specific shapes and dimensions, based on standardized norms. This document presents a process proposal for the elaboration of cylindrical and plate-shaped specimens, based on CRG granulated recycled rubber and 7 selected commercial binders. As a result of this investigation, the specimens obtained with polyurethane elastomeric sealant and polyurethane adhesive maintained their shape when the compression and the mould were removed. In a subsequent work, these specimens will be subjected to mechanical tests to determine the corresponding mechanical properties.
\end{abstract}

Keywords Binders, rubber, chemical properties, thermal properties, recycling.

* Corresponding author: nacari.marin@utp.ac.pa

\section{Introducción}

El dominio y la utilización de los materiales elastómeros, conocidos como hule o caucho, ha sido objeto de disputa e interés desde hace mucho tiempo, inclusive en la Segunda Guerra Mundial cuando este recurso tuvo implicaciones en el movimiento de fuerzas beligerantes en todo el mundo [1]. Sin embargo, su productividad representa un problema, debido a los niveles de contaminación que produce cuando el componente a base de caucho termina su vida útil.

Los neumáticos representan el 10\% de los desechos microplásticos que se localizan en los océanos de todo el planeta. Según la Unión Internacional para la Conservación de la Naturaleza (IUCN), el porcentaje de neumáticos aumentó en un 28\% durante el 2017 [2]. Según el Instituto Nacional de Estadística y Censo (INEC) del 2010 al 2018 el desecho de caucho en Panamá, tuvo un incremento del $10.91 \%$, lo cual se traduce en $305,285,130 \mathrm{~kg}$ de caucho desechado en 10 años, de los cuales 188, 872,604 kg provienen de los neumáticos. Los gobiernos se han visto involucrados en la necesidad de implementar regulaciones para disminuir la contaminación producida a través del uso de caucho reciclado en sus procesos industriales [3]. 
Para aprovechar los residuos del caucho, existen diferentes tipos de procesos como: Mecánicos, criomecánicos, termomecánicos, químico-mecánicos, y químicos, entre otros [4]. El proceso de trituración empleado dentro de los procesos mecánicos es el más utilizado debido a que el producto que se obtiene es fácil de aprovechar. Sus elevados costos y la contaminación que producen hacen de este proceso una opción poco viable para reciclar objetos de caucho; tal es el caso de los neumáticos [8].

Por lo antes expuesto, el reciclaje de caucho se ha incrementado en diferentes aplicaciones: fabricación de piezas y materiales de construcción ingenieriles como asfaltos, pavimentos y concretos, aisladores sísmicos y acústicos hasta implementos deportivos, calzado, pisos para áreas recreativas, pistas de juego y pisos de piscina [9].

Las probetas que se fabrican para ensayos de materiales no metálicos se preparan por arranque de viruta, por inyección y utilizando la técnica del prensado. De esta forma, el caucho se ha evaluado por diferentes técnicas que han permitido la definición de sus propiedades mecánicas y térmicas [5]. De allí que, para evaluar el caucho se requieran de probetas con formas y dimensiones específicas, basadas en normas estandarizadas.

El objetivo principal de este trabajo es presentar el proceso de fabricación de probetas de un material a base de caucho y aglomerantes comerciales, con el fin de obtener probetas cilíndricas y en forma de placas, para su posterior evaluación mediante pruebas mecánicas.

\section{Metodología}

La ejecución de este proyecto tomó en cuenta las probetas como aproximaciones a volúmenes definidos de cilindro y paralelepípedo, para las probetas cilíndricas y de placa respectivamente. Con ello, se obtienen los valores de masa de los productos que se necesitan para obtener una mezcla con unos porcentajes de volumen fijo.

$\mathrm{V}_{\mathrm{cil}}=\frac{\pi \cdot \mathrm{D}^{2} \cdot \mathrm{h}}{4}$

$\mathrm{V}_{\text {Placa }}=\mathrm{a}^{2} \cdot \mathrm{h}$

En las ecuaciones 1 y 2, D es el diámetro de la probeta, $\mathrm{h}$ es la altura, tanto para la probeta cilíndrica como para la probeta en forma de placa y es la medida de los lados en la probeta en forma de placa y se puede apreciar en la figura 1.

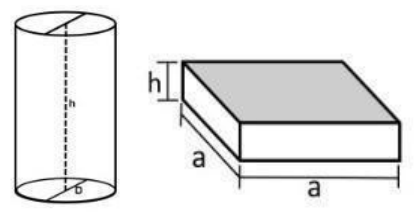

Figura 1. Volúmenes definidos de cilindro (izquierda) y paralelepípedo (derecha).
Se define el volumen total de la mezcla, mediante la siguiente relación:

$$
\begin{aligned}
& \mathrm{V}_{\mathrm{T}}=\sum \mathrm{V}_{\text {componentes }} \\
& \mathrm{V}_{\text {componente }}=\mathrm{X}_{\mathrm{i}} \cdot \mathrm{V}_{\mathrm{T}} \\
& \mathrm{V}_{\mathrm{T}}=\mathrm{X}_{1} \cdot \mathrm{V}_{\mathrm{T}}+\mathrm{X}_{2} \cdot \mathrm{V}_{\mathrm{T}}+\ldots+\mathrm{X}_{\mathrm{n}} \cdot \mathrm{V}_{\mathrm{T}}
\end{aligned}
$$

Donde $\mathrm{V}_{\mathrm{T}}$ es el volumen de la mezcla dentro de la probeta (cilindro o de placa), $X_{\mathrm{i}}$ son los porcentajes de los componentes de la mezcla, concerniente al volumen total de la mezcla. Después se utiliza la relación entre densidad, masa y volumen para despejar la masa de las ecuaciones:

$$
\rho=\frac{m}{V}
$$

$\mathrm{X}_{\mathrm{i}} \cdot \mathrm{V}_{\mathrm{T}}=\mathrm{V}_{\text {comp }}$

$\mathrm{X}_{\mathrm{i}} \cdot \mathrm{V}_{\mathrm{T}}=\frac{\mathrm{m}_{\text {comp }}}{\rho_{\text {comp }}}$

$\mathrm{m}_{\text {comp }}=\mathrm{X}_{\mathrm{i}} \cdot \mathrm{V}_{\mathrm{T}} \cdot \rho_{\text {comp }}$

Ya se conoce $\mathrm{M}$ como el valor de la masa y $\mathrm{V}$ el volumen obtenido con las ecuaciones 1 o 2 , depende de la probeta que se vaya a realizar. De esta manera, con la ecuación (5), se obtienen las cantidades de masa $\left(\mathrm{m}_{\text {comp }}\right)$ necesarias para obtener una mezcla heterogénea volumétrica, con cualquier aglomerante en cualquier proporción. $X_{\mathrm{i}}$ corresponde a la variable que determina el porcentaje de aglomerante, $V_{T}$ el volumen final de la mezcla dentro de la probeta y $\rho_{\text {comp la }}$ densidad del aglomerante.

\subsection{Materiales}

El caucho es un componente con propiedades elásticas y es resistente a ácidos y sustancias alcalinas. Repele el agua, aísla la temperatura y la electricidad, resiste al estiramiento y conserva su propiedad de flexibilidad, incluso en condiciones de frío. Es capaz de disolverse ante la presencia del benceno y algunos hidrocarburos [6].

El proceso de fabricación de las probetas cilíndricas y en forma de placas, se utilizó como materia prima caucho reciclado granulado (CRG). Este material se suele utilizar como base para las canchas de fútbol sintéticas. Se utilizaron diferentes tipos de aglomerantes, que se mencionan a continuación: LANCO SiliconFlex Elastomérico Acrílico Siliconizado, LANCO PolyFlex Sellador Elastomérico de Poliuretano, LANCO Siliconizer Ultra White Elastomeric Crack Filler, LANCO Masilla Acrílica Multiusos, Adhesivo de 
Poliuretano PLASTIPOL 444, LANCO Urethane-WB, LANCO Grip Bond 2 y resina de fibra de vidrio.

En la selección de estos materiales se partió de la premisa que el producto final debía tener propiedades elastoméricas. Por ende, se seleccionaron distintos selladores, adhesivos y masillas comerciales, que entre sus aplicaciones se encuentra la adhesión y reparación de superficies. El aglomerante debía ser capaz de solidificarse a temperatura ambiente.

A través de los experimentos que se llevaron a cabo se buscó comprobar si al utilizar estos materiales como aglomerantes, mantenían propiedades de resistencia y elasticidad. En las referencias consultadas, el caucho se ha mezclado con: concreto [10], asfalto [11], betún asfáltico [12] $\mathrm{y}$ no se ha encontrado un material que se constituya en una mezcla de caucho y selladores, adhesivos o masillas comerciales. Se utilizó arena de sílice tamizada por un filtro de $3 \mathrm{~mm}$ como un componente añadido al aglomerante, para reducir la cantidad de aglomerante industrial en la mezcla y aportar mayor densidad en la mezcla.

\subsection{Métodos}

Como parte de este trabajo, se confeccionaron dos tipos de moldes. Para las probetas cilíndricas, se utilizó un tubo de PVC de diámetro interno de 2" y 4" de largo, un tapón de madera removible de 2.0" de diámetro y aproximadamente 1.0" de espesor y, por último, un tapón de PVC de 2.0" de diámetro (ver Figura 2). Cada molde solo puede ser utilizado una vez. Y las probetas en forma de placas se construyeron con tablas siguiendo las normas ASTM-C177, que es un método de prueba para medir la conductividad térmica de los materiales utilizando una configuración de placa con las siguientes medidas: $300 \mathrm{~mm}$ de ancho, $300 \mathrm{~mm}$ de largo y $15 \mathrm{~mm}$ de espesor [7].

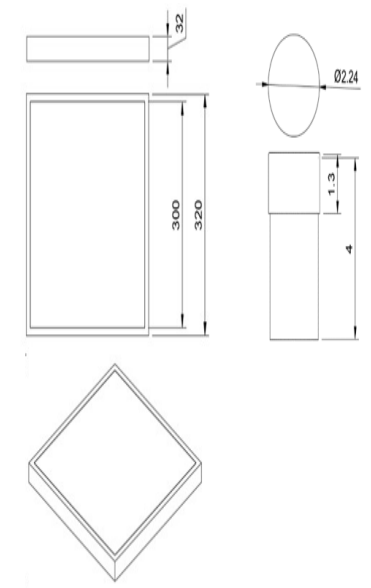

Figura 2. Moldes para las probetas.
A continuación, se detalla la metodología utilizada para la fabricación de las probetas cilíndricas y en forma de placas:

- Se calcularon las cantidades de CRG y de aglomerante por peso con respecto a su porcentaje del volumen total. Para ello, se utilizó la fórmula (5) con lo que se confeccionó la tabla 1.

Tabla 1. Datos de densidades y masas de aglomerante utilizado en cada probeta

Caucho

Arena

\begin{tabular}{|c|c|}
\hline Densidad $\left(\mathbf{k g} / \mathbf{m}^{\mathbf{3}}\right)$ & Densidad $\left(\mathbf{k g} / \mathbf{m}^{\mathbf{3}}\right)$ \\
\hline 957 & 1682 \\
\hline
\end{tabular}

\begin{tabular}{|c|c|c|c|c|}
\hline \multicolumn{5}{|c|}{ Probeta Cilíndrica } \\
\hline Aglomerante & $\begin{array}{c}\text { Densidad } \\
\left(\mathrm{kg} / \mathrm{m}^{3}\right)\end{array}$ & $\begin{array}{c}\text { Proporción } \\
\text { de la } \\
\text { Composición }\end{array}$ & $\begin{array}{c}\text { Masa } \\
(\mathrm{g})\end{array}$ & Ref \\
\hline $\begin{array}{c}\text { LANCO } \\
\text { SiliconFlex }\end{array}$ & 1270.77 & $60 \%-40 \%$ & 111.22 & [13] \\
\hline $\begin{array}{l}\text { LANCO } \\
\text { PolyFlex }\end{array}$ & 1014.86 & $75 \%-25 \%$ & 55.540 & {$[13]$} \\
\hline $\begin{array}{c}\text { LANCO } \\
\text { Siliconizer }\end{array}$ & 1015.38 & $60 \%-40 \%$ & 88.86 & [13] \\
\hline $\begin{array}{c}\text { Resina de } \\
\text { Fibra de } \\
\text { Vidrio }\end{array}$ & 2580 & $75 \%-25 \%$ & 141.12 & [14] \\
\hline $\begin{array}{c}\text { Resina de } \\
\text { Fibra de } \\
\text { Vidrio } \\
\end{array}$ & 2580 & $60 \%-40 \%$ & 225.79 & [14] \\
\hline $\begin{array}{l}\text { Adhesivo de } \\
\text { Poliuretano }\end{array}$ & 930.6 & $60 \%-40 \%$ & 81.44 & $*$ \\
\hline $\begin{array}{c}\text { LANCO } \\
\text { Urethane-WB }\end{array}$ & 1301.1 & $60 \%-40 \%$ & 113.87 & {$[13]$} \\
\hline $\begin{array}{c}\text { LANCO Grip } \\
\text { Bond } 2\end{array}$ & 881.32 & $60 \%-40 \%$ & 77.13 & {$[13]$} \\
\hline \multicolumn{5}{|c|}{ Probeta de Placa } \\
\hline Aglomerante & $\begin{array}{c}\text { Densidad } \\
\left(\mathrm{kg} / \mathrm{m}^{3}\right)\end{array}$ & $\begin{array}{c}\text { Proporción } \\
\text { de la } \\
\text { composición }\end{array}$ & $\begin{array}{c}\text { Masa } \\
(\mathrm{g})\end{array}$ & Ref \\
\hline $\begin{array}{c}\text { Fibra de } \\
\text { Vidrio }\end{array}$ & 2580 & $50 \%-50 \%$ & 2322 & {$[14]$} \\
\hline $\begin{array}{c}\text { LANCO } \\
\text { SiliconFlex }\end{array}$ & 1270.77 & $60 \%-40 \%$ & 912.95 & {$[13]$} \\
\hline $\begin{array}{c}\text { LANCO } \\
\text { PolyFlex } \\
\text { Sellador }\end{array}$ & 1014.86 & $60 \%-40 \%$ & 730.69 & [13] \\
\hline
\end{tabular}

*Datos medidos experimentalmente

- Las cantidades calculadas se virtieron en un recipiente, para luego realizar la mezcla hasta conseguir que fuera heterogénea y uniforme. 
Marín (et al): Propuesta de proceso para la fabricación de probetas a base de caucho reciclado granulado

- Después se procedió a colocar la mezcla en moldes y se comprimió. En este caso se utilizó una prensa hidráulica y una estructura de hierro. También se puede utilizar una prensa sargento para lograr la compresión. Es importante que todas las probetas reciban una compresión lo más cercana posible entre ellas. En este caso, se determinó un número de compresión por palanca, el cual fue de 5 veces.

- Se dejó secar cada mezcla el tiempo estipulado en las instrucciones del fabricante de cada aglomerante. En la tabla 2, se detallan los tiempos de secado de cada aglomerante:

Tabla 2. Tiempos de secado de los aglomerantes

\begin{tabular}{|c|c|c|}
\hline Aglomerante & Tiempo de secado & Ref. \\
\hline LANCO SiliconFlex & 24 horas & {$[13]$} \\
\hline LANCO PolyFlex & 6 horas & {$[13]$} \\
\hline LANCO Siliconizer & 24 horas & {$[13]$} \\
\hline Resina de Fibra de Vidrio & +48 horas & {$[14]$} \\
\hline Adhesivo de Poliuretano & 3 horas & $*$ \\
\hline LANCO Urethane-WB & 24 horas & {$[13]$} \\
\hline LANCO Grip Bond 2 & 30 minutos & {$[13]$} \\
\hline
\end{tabular}

*Dato obtenido en la etiqueta del producto.

- Se procedió a extraer el compuesto resultante. En el caso de las probetas cilíndricas, se debe retirar el tapón de madera y cortar el tubo de PVC. Para las probetas en forma de placa, solo se tiene que retirar el plástico.

- Se inspeccionó si el aglomerante se había secado por completo o si se debe proporcionar más tiempo de secado.

\section{Resultados}

Si bien es cierto que el compuesto principal de la probeta es el CRG, la selección del aglomerante tiene un efecto en las propiedades del producto final. Por esta razón, a pesar que el proceso fue el mismo para todos los casos, los resultados fueron diferentes. En las siguientes secciones, se muestran los resultados cualitativos obtenidos durante la elaboración de las probetas cilíndricas y las probetas en forma de placas.

En la tabla 3, se presentan los detalles de cada una de las probetas cilíndricas, acompañados de una imagen y de observaciones generales en cada caso. En general, se puede comentar la falta de adhesión completa entre el CRG y el aglomerante en la mayoría de los casos.
Tabla 3. Probetas cilíndricas, según porcentaje de compuestos mezclados

\begin{tabular}{|c|c|c|c|}
\hline Materiales & $\begin{array}{l}\text { Proporción } \\
\text { de la } \\
\text { composición }\end{array}$ & Imagen & Observación \\
\hline $\begin{array}{l}\text { CRG- } \\
\text { Resina de } \\
\text { Fibra de } \\
\text { Vidrio }\end{array}$ & $75 \%-25 \%$ & & $\begin{array}{l}\text { No se presentó } \\
\text { adhesión entre el } \\
\text { CRG y el } \\
\text { aglomerante. No } \\
\text { mantuvo la forma } \\
\text { cilíndrica al } \\
\text { retirar el molde. }\end{array}$ \\
\hline $\begin{array}{l}\text { CRG- } \\
\text { Resina de } \\
\text { Fibra de } \\
\text { Vidrio }\end{array}$ & $60 \%-40 \%$ & & $\begin{array}{l}\text { No se presentó } \\
\text { adhesión entre el } \\
\text { CRG y el } \\
\text { aglomerante. No } \\
\text { mantuvo la forma } \\
\text { cilíndrica al } \\
\text { retirar el molde. }\end{array}$ \\
\hline $\begin{array}{c}\text { CRG- } \\
\text { Siliconflex }\end{array}$ & $60 \%-40 \%$ & & $\begin{array}{lr}\text { El CRG } & \text { se } \\
\text { adhirió } & \text { al } \\
\text { aglomerante. No } & \text { Nantuvo la forma } \\
\text { cilíndrica al } & \text { atirar el molde. }\end{array}$ \\
\hline $\begin{array}{c}\text { CRG- } \\
\text { PolyFlex }\end{array}$ & $75 \%-25 \%$ & & $\begin{array}{l}\text { Se presentó } \\
\text { adhesión entre el } \\
\mathrm{CRG} \text { y el } \\
\text { aglomerante. } \\
\text { Mantuvo la forma } \\
\text { cilíndrica al } \\
\text { retirar el molde. }\end{array}$ \\
\hline $\begin{array}{c}\text { CRG- } \\
\text { Plastipol } \\
444\end{array}$ & $60 \%-40 \%$ & & $\begin{array}{l}\text { Se presentó } \\
\text { adhesión entre el } \\
\text { CRG y el } \\
\text { aglomerante.El } \\
\text { aglomerante se } \\
\text { expandió al } \\
\text { retirar el molde, } \\
\text { con lo que se } \\
\text { obtuvo una forma } \\
\text { irregular. }\end{array}$ \\
\hline $\begin{array}{l}\text { CRG- } \\
\text { Urethane- } \\
\text { WB }\end{array}$ & $60 \%-40 \%$ & & $\begin{array}{l}\text { La mezcla no se } \\
\text { secó por } \\
\text { completo, } \\
\text { transcurrido el } \\
\text { tiempo de secado } \\
\text { sugerido por el } \\
\text { fabricante. }\end{array}$ \\
\hline
\end{tabular}


Marín (et al): Propuesta de proceso para la fabricación de probetas a base de caucho reciclado granulado

\begin{tabular}{|c|c|c|}
\hline $\begin{array}{c}\text { CRG- } \\
\text { Arena- } \\
\text { PolyFlex }\end{array}$ & $\begin{array}{c}75 \%-10 \%- \\
15 \%\end{array}$ & $\begin{array}{ll}\text { Se presentó } \\
\text { adhesión entre los } \\
\text { componentes y se } \\
\text { observa poca } \\
\text { compactación. } \\
\text { Mantiene ru } \\
\text { forma cilíndrica } \\
\text { al retirar el } \\
\text { molde. } \\
\text { observan } \\
\text { propiedades } \\
\text { elásticas. }\end{array}$ \\
\hline $\begin{array}{c}\text { CRG- } \\
\text { Arena- } \\
\text { PolyFlex }\end{array}$ & $\begin{array}{c}60 \%-20 \%- \\
20 \%\end{array}$ & $\begin{array}{ll}\text { Se mostró } \\
\text { adhesión entre los } \\
\text { componentes. } \\
\text { Mantiene ru } \\
\text { forma cilíndrica } \\
\text { al retirar el } \\
\text { molde. r Se } \\
\text { observan r } \\
\text { propiedades } \\
\text { elásticas. }\end{array}$ \\
\hline $\begin{array}{c}\text { CRG- } \\
\text { Arena- } \\
\text { Plastipol } \\
444\end{array}$ & $\begin{array}{c}60 \%-20 \%- \\
20 \%\end{array}$ & $\begin{array}{l}\text { Se demostró } \\
\text { adhesión entre los } \\
\text { componentes y } \\
\text { mantuvo su } \\
\text { forma cilíndrica } \\
\text { al retirar el } \\
\text { molde. Al tacto } \\
\text { parece ser muy } \\
\text { duro y resistente. }\end{array}$ \\
\hline $\begin{array}{c}\text { CRG-Grip } \\
\text { Bond }\end{array}$ & $60 \%-40 \%$ & $\begin{array}{l}\text { No se presentó } \\
\text { adhesión entre el } \\
\text { CRG y el } \\
\text { aglomerante. No } \\
\text { mantuvo la forma } \\
\text { cilíndrica al } \\
\text { retirar el molde. } \\
\text { La mezcla no se } \\
\text { seca } \\
\text { completo, por } \\
\text { transcurrido el } \\
\text { tiempo de secado } \\
\text { sugerido por el } \\
\text { fabricante. }\end{array}$ \\
\hline
\end{tabular}

Se fabricaron probetas en forma de placas, las cuales arrojaron resultados muy similares a las probetas cilíndricas. En la tabla 4, se presentan los detalles de las 3 placas fabricadas.
Tabla 4. Probetas en forma de placa, según porcentaje de compuestos

\begin{tabular}{|c|c|c|l|}
\hline Materiales & Cantidades & Imágen & \multicolumn{1}{|c|}{ Observación } \\
\hline $\begin{array}{c}\text { CRG- } \\
\text { Resina de } \\
\text { Fibra de } \\
\text { Vidrio }\end{array}$ & $50 \%-50 \%$ & $\begin{array}{l}\text { lo hubo } \\
\text { adhesión y la } \\
\text { forma se deshacía } \\
\text { con facilidad. }\end{array}$ \\
\hline $\begin{array}{c}\text { CRG- } \\
\text { SILICONF } \\
\text { LEX }\end{array}$ & $60 \%-40 \%$ & & $\begin{array}{l}\text { Se adherió con el } \\
\text { CRG, pero al al } \\
\text { sacarla del } \\
\text { molde, perdía la } \\
\text { forma. }\end{array}$ \\
\hline CRG- & $60 \%-40 \%$ & $\begin{array}{l}\text { Hubo adhesión } \\
\text { con el CRG y } \\
\text { mantuvo su } \\
\text { forma, luego de } \\
\text { sacarla del } \\
\text { molde. }\end{array}$ \\
\hline
\end{tabular}

\section{Discusión}

Los resultados obtenidos corresponden a observaciones y análisis cualitativos. Este estudio ha permitido definir una metodología de fabricación de probetas cilíndricas y en forma de placas a base de caucho granulado y dos selladores comerciales. A su vez, ha permitido identificar los selladores que, al combinarse con el caucho granulado, mantienen la forma cilíndrica o de placa, según el caso.

Entre los resultados obtenidos se debe destacar que los únicos aglomerantes que mantuvieron la forma del molde, resultando en un material compacto, tanto en forma cilíndrica como de placa, fueron los aglomerantes LANCO Polyflex Sellador Elastomérico de Poliuretano y Adhesivo de Poliuretano PLASTIPOL 444 (ambos a base de poliuretano). $\mathrm{Al}$ inspeccionar estos materiales, se puede apreciar la presencia del CRG en la mezcla, así como una superficie medianamente áspera al tacto. Sobre la superficie de la probeta del aglomerante LANCO PolyFlex se observaron propiedades elásticas, mientras que en el caso del Adhesivo de Poliuretano PLASTIPOL 444 se observó una mezcla con una dureza mayo. Entonces, para poder demostrar esta premisa se debe someter a las respectivas pruebas mecánicas.

Cuando se añadió la arena en la mezcla se obtuvieron probetas más densas al tacto con ambos aglomerantes a simple vista.

En el caso de las placas, al flexionarlas ligeramente (ver figura 3) y liberarlas, recuperan su posición original sin deformarse permanentemente. Cabe señalar, que la placa 
presenta diferentes espesores, debido a una distribución no controlada de la mezcla en el molde correspondiente.

Bajo estos resultados se puede suponer que el poliuretano y la arena tienen un efecto positivo en la mezcla, pues ambos componentes en las proporciones ensayadas se obtuvieron probetas con mejor compactación y aglomeración con el caucho. Aunque los resultados presentados son cualitativos, este estudio muestra un material que presenta propiedades elásticas que deben ser evaluadas, con el fin de identificar las aplicaciones potenciales del material. En este sentido, y de acuerdo a referencias consultadas, el caucho puede ser mezclado con otros materiales [10][11][12], en la búsqueda de soluciones para aplicaciones en donde la recuperación elástica es un tema importante.

El poliuretano es un compuesto que se utiliza como adhesivo, capaz de unir materiales, aportando tenacidad y resistencia a un material al secarse. En Paredes et Urquizo, 2021, se presenta un estudio de caracterización del comportamiento mecánico de un material compuesto a partir de matriz de poliuretano, reforzado con gránulos de caucho reciclado y fibras, demostrando la mejora en las propiedades al combinar estos materiales [15].

La experimentación realizada ayuda a verificar que el poliuretano logra una adhesión con el caucho, permitiendo la obtención de formas, de acuerdo al molde empleado, que permitió descartar aglomerantes que por sus características y antecedentes en la industria se consideró que podían cumplir con los requerimientos para llevar a cabo la fabricación del nuevo material. En Fernández, 1991, se presentó el proceso de unión de un adhesivo poliuretano y caucho, lo que apoya los resultados obtenidos en la presente investigación [16].

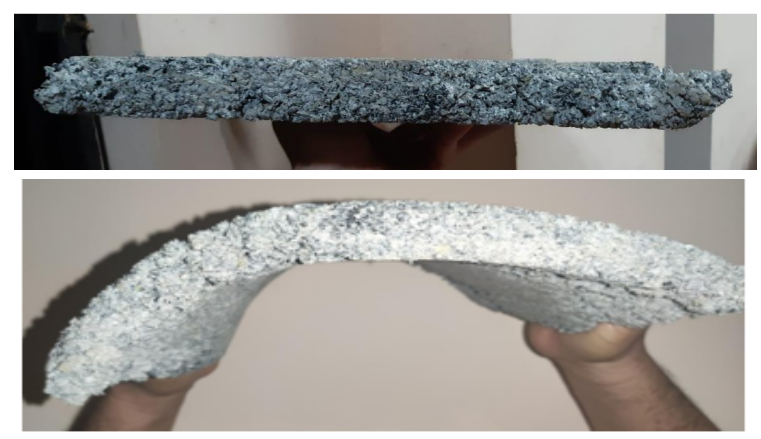

Figura 3. Placa a base de caucho SBR y PolyFlex recta y flexionada.

El resto de las probetas no lograron una adhesión y compactación entre el CRG y el aglomerante correspondiente, durante su proceso de elaboración, por lo que durante la extracción del molde se separaban los materiales. En algunos casos, los gránulos de CGR se despegaban entre ellos, aun cuando se encontraban inmersos en el aglomerante. Esto puede deberse principalmente a los componentes del aglomerante comercial, a diferencia de los aglomerantes que contienen poliuretano que si lograron mantener la forma al ser retirados del molde.

Las probetas en forma de placa que se confeccionaron utilizando masilla acrílica y resina de fibra de vidrio como aglomerante, no presentaron una adhesión entre el CRG y los aglomerantes mencionados (ver imágenes presentadas en las tablas 3 y 4). En estos casos, se puede comentar que no resistieron el proceso de desmolde sin romperse o agrietarse. La placa con resina de fibra de vidrio no logró unir los granos de forma satisfactoria. La masilla acrílica logró unirse con el caucho granulado y la mezcla se mantuvo poco compacta, lo que ocasionó su separación.

En este trabajo se evitó agregar mayor cantidad de aglomerante con respecto a las cantidades de CRG, debido a que esta investigación se centra en el proceso de elaboración de un material a base de caucho reciclado como su principal componente, así como la verificación del proceso de fabricación planteado para la elaboración de un nuevo material a base de un material que normalmente es considerado como desecho y su potencial reutilización en aplicaciones de ingeniería.

\section{Conclusiones}

El caucho es un material útil y con propiedades físicas muy ventajosas. El principal problema de utilizar caucho es que se torna un contaminante a nivel visual y a nivel de microplásticos, afectando gravemente los ecosistemas acuáticos.

En esta investigación se presentó una metodología para la elaboración de probetas cilíndricas y placas a base de caucho reciclado granulado y aglomerantes (selladores) comerciales. De los siete aglomerantes estudiados, solo los aglomerantes LANCO Polyflex Sellador Elastomérico de Poliuretano y Adhesivo de Poliuretano PLASTIPOL 444, presumiblemente, presentan adhesión y compactación con los gránulos de caucho reciclado, por lo que en la etapa siguiente de esta investigación se espera realizar pruebas mecánicas que permitan determinar las propiedades de forma cuantitativa.

La indagación sienta las bases para el estudio de nuevos materiales a base de caucho reciclado. La fabricación de probetas a partir de caucho reciclado es el primer paso en el proceso de medir y probar las distintas propiedades que estos materiales pueden presentar, con el objetivo de identificar aplicaciones potenciales futuras.

Se recomienda caracterizar el producto final realizándole pruebas mecánicas y de conductividad térmica y eléctrica. Por lo que, se recomienda automatizar el proceso de mezclado o utilizar una herramienta que permita mezclar los dos compuestos, evitando así el contacto directo con la piel. 
Así mismo, se sugiere confeccionar un nuevo molde que se pueda reutilizar y obtener más resultados con menor costo y residuos sólidos.

En investigaciones futuras se recomienda examinar la relación entre caucho, arena y aglomerante para determinar cuál es la proporción óptima de cada uno de estos tres componentes, con el fin de obtener mejores resultados.

\section{AGRADECIMIENTOS}

Reconocemos al Taller de Mecánica Biker Solution by Benza, por prestar su instalación y herramientas para la fabricación de las probetas.

\section{REFERENCIAS}

[1] C. Domínguez, "Geografía política del caucho durante la Segunda Guerra Mundial" 1995. [Online]. Available: file:///C:/Users/anabe/Downloads/Dialnet-

GeografiaPoliticaDelCauchoDuranteLaSegundaGuerraMu6581681.pdf [Feb. 7, 2021]

[2] T. Root, "National Geographic" Sept. 2019. [Online]. Available: https://www.nationalgeographic.es/medioambiente/2019/09/neumaticos-gran-fuente-contaminacionplastico [Feb. 7, 2021]

[3] G. Peláez, S. Velásquez y D. Giraldo, "Aplicaciones del Caucho Reciclado: una revisión de la literatura." Ciencia e Ingeniería Neogranadina, vol 27, num. 2, 2017. [Online]. Available: https://www.redalyc.org/jatsRepo/911/91150559002/html/inde x.html\#redalyc_91150559002_ref69 [Feb. 7, 2021]

[4] E. Abraham, B. Cherian, P. Elbi, L. Pothen y S. Thomas, "Recent Advances in the Recycling of Rubber Waste." 2011. [Online]. Available: https://www.researchgate.net/publication/282235405_Recent_ advances_in_the_recycling_of_rubber_waste [Feb. 7, 2021]

[5] "Ensayo de tracción-Práctica 2." Bloque 2, pp. 1-2.

[6] C. Iglesias, "Materiales: El Caucho." monografías.com, [Online]. Available: https://www.monografias.com/trabajos72/materialescaucho/materiales-caucho.shtml [Feb. 7, 2021]

[7] R. Ruiz, R. Canto y L. Lira, "Equipo para la medición de la conductividad térmica de materiales homogéneos y heterogéneos." Simposio de Metrología, pp. 6-10, 2014.

[8] M. Forrest, "Recycling and Re-use of Waste Rubber." Shoropshire: Smithers Information, 2014.

[9] M. Recycling, "Micor Recycling." [Online]. Available: http://www.micorrecycling.com/knowledge.php. karin.fq.uh.cu/acc/2016/CIENCIAS_TECNICAS/032/New/.../ Parte\%20V.pdf. [Jan. 31, 2021]

[10] George B. Way, Han Zhu, Kamil E. Kaloush, "Properties of Crumb Rubber Concrete." The 2005 Annual Meeting of the Transportation Research Board, 2004 .

[11] Omprakash, V. Chandralega, V. Suganpriya, "Study of Behaviour of Bitumen Modified with Crumb Rubber." International Journal of Engineering Research \& Technology (IJERT), vol. 5, Nº5, 2016.

[12] Ahmed R. Suleiman, Moncef L. Nehdi, Saud A. Alfayez, "Recycling Tire Rubber in Asphalt Pavements: State of Art." MDPI, vol. 12, 2020.
[13] Anónimo, "Wikipeda" [Online]. Available: https://es.wikipedia.org/wiki/Fibra_de_vidrio [Feb. 7, 2021]

[14] LANCO, "LANCO Paints." [Online]. Available: https://www.lancopaints.com/america-central/es/ [Feb. 7, 2021]

[15] J. Paredes, G. Urquizo, "Caracterización del comportamiento mecánico de un material compuesto a partir de matriz de poliuretano monocomponente, reforzado con gránulo de caucho reciclado y fibra de abacá como material alternativo, para aplicaciones industriales." 2021. [Online]. Available: http://repositorio.uta.edu.ec/handle/123456789/32149 [Feb. 7, 2021]

[16] J. C. Fernández García, "Análisis del proceso de adhesión en el sistema caucho sintético-adhesivo de poliuretano." 1991. [Online]. Available: http://rua.ua.es/dspace/handle/10045/3492 [Feb. 7, 2021] 